\title{
Ankylosing Spondylitis - the changing role of imaging
}

\author{
Zoe Ash • Helena Marzo-Ortega
}

Published online: 22 June 2012

(C) ISS 2012

\section{Introduction}

Ankylosing spondylitis (AS) is the disease prototype within a heterogeneous group of inflammatory arthritides known collectively as the seronegative spondyloarthropathies (SpA). The other subtypes as originally described by Moll and Wright are psoriatic arthritis, reactive arthritis and inflammatory bowel disease associated arthritis. The pathogenic hallmark of these diseases is inflammation at the entheses; the sites of attachment of ligament, fascia or tendon insertions into bone. This process affects primarily the axial skeleton, including the spine and sacroiliac joints. Extra-articular features of the disease can include uveitis, aortitis and inflammatory bowel disease. In the majority of cases AS affects individuals in their early twenties and thirties, causing long term pain and disability and leading to work instability and decreased quality of life. Early mortality has been reported, associated mainly with an increased risk of cardiovascular morbidity.

\footnotetext{
Z. Ash $\cdot$ H. Marzo-Ortega $(\triangle)$

Division of Rheumatic and Musculoskeletal Disease, Leeds Institute of Molecular Medicine, University of Leeds,

2nd Floor, Chapel Allerton Hospital, Chapeltown Road, Leeds LS7 4SA, UK

e-mail: medhmo@leeds.ac.uk

Z. Ash

e-mail: Z.Ash@leeds.ac.uk

Z. Ash $\cdot$ H. Marzo-Ortega

NIHR Leeds Musculoskeletal Biomedical Research Unit, Leeds

Teaching Hospitals NHS Trust,

Leeds, UK
}

\section{MRI in the diagnosis and classification of AS}

One of the biggest handicaps in the study of these diseases has been the lack of diagnostic features and overt clinical signs of disease. The modified New York criteria (mNYC) have been the traditional tool to diagnose AS, and continue to be widely used [1]. These criteria rely on the detection of radiographic sacroiliitis, in association with clinical symptoms and signs such as reduction of lumbar movements or chest expansion to make a diagnosis. The inclusion of radiographic sacroiliitis was based on the traditionally held belief that the sacroiliac joints are the anatomic area most frequently affected in the early stages and, hence, they are the most sensitive area to detect the characteristic bony damage in AS. However one of the most important advances in the clinical understanding of AS has been the recognition of the significant time delay between the onset of symptoms, i.e., inflammatory back pain, and the development of radiographic evidence of sacroiliac joint damage, which can take an average of eight to ten years to become detectable. Furthermore, recent studies have shown that patients in the early stages of disease, prior to the appearance of radiographic sacroiliitis, have just as much pain, stiffness and functional limitation as those with established or radiographic AS [2]. This has led to increasing interest in the use of magnetic resonance (MR) imaging to detect changes at the sacroiliac joint or spine at an earlier stage.

The unique imaging capabilities of MR that allow for the visualization of not only bone but soft tissue have contributed to significant advances in the understanding of disease pathogenesis in SpA. The diagnostic findings visible on radiography correspond to lesions representative of a chronic process of structural bony damage, such as sclerosis, erosions, joint pseudo-widening or ankylosis, which constitute an irreversible finding. By contrast, MRI can 
identify acute oedematous bony lesions which have been shown to correlate at the histological level to an osteitis, confirming the inflammatory nature of these lesions in SpA. These bone marrow oedema (BMO) lesions, although not specific for $\mathrm{SpA}$, represent the most sensitive diagnostic feature particularly in the early stages and have been shown to correlate with patients' symptoms. Furthermore, recent work has confirmed the predictive value of an abnormal MRI in a group of patients with early inflammatory back pain. The combination of severe BMO lesions (according to the Leeds MRI Scoring System) and a positive HLA B27 each gave a sensitivity and specificity of $77 \%$ for the subsequent development of radiographic sacroiliitis [3]. These findings were confirmed in a separate prospective study.

The value of MRI in the early recognition of sacroiliitis has been recognized by the Assessment of Spondyloarthritis International Society (ASAS) with the development of new classification criteria for axial SpA [4]. These classification criteria allow for the recognition of sacroiliitis not only on radiographs but also on MRI. To aid with the implementation of the criteria, a definition of what constitutes a positive sacroiliitis signal in $\mathrm{SpA}$ was recently proposed, formulated by consensus agreement by the ASAS-OMERACT (Outcome Measures in Rheumatology) MRI group of expert radiologists and rheumatologists [5]. This definition is based on the finding of BMO on a short TI inversion recovery (STIR) sequence or osteitis on a gadolinium enhanced T1weighted fat suppressed MRI sequence in the subchondral or periarticular bone marrow of the sacro-iliac joint. According to this, an MRI is considered "highly suggestive of

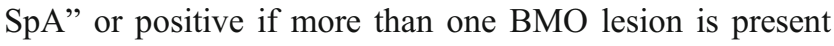
on a single slice or if one lesion is present on two consecutive slices. A recent study has shown that this definition appears to be of diagnostic utility in early SpA although it has limited prognostic value for the development of radiographic progression [6].

\section{MRI as a prognostic biomarker}

Recent studies have demonstrated that MRI determined BMO lesions [7] and an elevated $\mathrm{C}$ reactive protein (CRP) are the main biomarkers for prediction of response to tumour necrosis factor alpha (TNF $\alpha$ ) blocking treatment. This has led to the increasing use of MRI in the clinical setting to target treatment to those patients with active disease.

\section{Which areas should be imaged?}

Imaging to assess disease activity in the axial skeleton should include not only the sacroiliac joints but also the whole spine. There are a number of reasons for this. Firstly, spinal inflammation (bone marrow oedema) is seen most frequently in the thoracic spine regardless of clinical symptomatology and affects frequently not only the vertebral bodies but the posterior elements. These lesions have been found to be highly specific for SpA and AS. One small study found inflammation isolated to the posterior elements of the spine on MRI in 8 of 11 patients with a short duration of inflammatory back pain (median duration four months) [8]. It has been proposed that for diagnostic evaluation and to assess disease activity, imaging assessment should include sagittal images that extend to the lateral edges of all thoracic vertebrae. Previously, scoring systems for radiographs of the spine in AS excluded the thoracic spine due to the problems of overlying tissue complicating interpretation and thus MRI offers a significant advantage in being able to study this area.

\section{Current treatment options}

There remains an important emphasis on education, physiotherapy and regular exercise in the treatment of AS. Nonsteroidal anti-inflammatory drugs (NSAIDs) can give excellent symptom relief but caution must be exercised with their long term use given their potential side effects. There is little evidence for the efficacy of standard disease modifying treatments such as methotrexate in managing the axial symptoms of AS.

Following on from the successful use of TNF $\alpha$ blocking agents in rheumatoid arthritis (RA), the role of TNF $\alpha$ in the pathogenesis of AS has been confirmed and the TNF $\alpha$ blocking agents adalimumab, etanercept, golimumab and infliximab have demonstrated efficacy in the management of AS [9]. Double blind placebo controlled randomised clinical trials (RCTs) followed by long term extensions have shown improvements in the symptoms of AS including pain, fatigue and morning stiffness, improvements in quality of life measures and spinal mobility measures along with reductions in the CRP. Furthermore, the same level of efficacy has been shown in the presence of spinal fusion in advanced disease.

\section{New strategies for treatment}

The next logical step is the consideration of treatment before irreversible damage has occurred. In contrast to RA where the main pathogenic outcome of uncontrolled inflammation is joint destruction, AS is a disease of new bone formation, with the formation of syndesmophytes contributing to the gradual loss of spinal mobility. Waiting for patients to fulfil the mNYC prior to offering effective therapy may cause 
distress, loss of function and potentially loss of employment, but also allows irreversible structural damage to occur.

A sub-analysis of two RCTs in AS has shown short disease duration to be a predictor of response to TNF $\alpha$ blocking therapy [10]. Two studies of TNF $\alpha$ blocking therapy in patients with non-radiographic early AS have shown excellent response, with ASAS partial remission rates up to $56 \%[11,12]$. These rates are much higher than those seen in the original RCTs. However, as the initial RCTs recruited only patients with established AS, in many countries the use of $\mathrm{TNF} \alpha$ blocking therapy is still restricted to patients fulfilling the mNYC, with moderate to severe disease.

\section{Searching for a possible link between inflammation and new bone formation}

Unlike in RA or psoriatic arthritis, where $\mathrm{TNF} \alpha$ blocking therapy has demonstrated impressive results in the inhibition of radiographic progression, this has not been shown in AS. The mechanisms for new bone formation in AS remain elusive and different theories have been proposed to explain this paradoxical effect. One of the main difficulties when interpreting the data from the clinical trials may relate to the short follow up times which average two years in the majority of cases. Other possible explanations are the fact that new bone formation is a late consequence of inflammation and thus may have a long lag time to see effect, the possibility of low grade ongoing inflammation even in those with clinical improvement on TNF $\alpha$ blocking therapy and the suggestion that $\mathrm{TNF} \alpha$, in the presence of active inflammation, reduces new bone formation. Thus once inflammation improves on TNF $\alpha$ blocking treatment, the "brake" is released and repair and new bone formation can proceed [13]. This possible link with inflammation is supported by a recent study suggesting that syndesmophyte formation is more frequent in vertebral corners in which a previous inflammatory lesion has resolved [14]. A second study found that fatty vertebral corner lesions, thought to be a post-inflammatory finding, predate syndesmophyte formation in affected spinal units [15]. This whole area remains of considerable interest and developments may have an effect on future treatment strategies. In fact, the only treatment shown to reduce radiographic progression is the continuous use of NSAIDs in comparison to "on demand" use [16].

\section{Conclusion}

The advent of effective treatments for AS has improved the outlook for patients at every stage of their disease process. MRI allows for an early diagnosis, thus permitting treatment before spinal fusion occurs. Still, much needs to be understood about the possible link between inflammation and new bone formation and fusion since TNF $\alpha$ blocking therapies do not appear to halt radiographic progression. Larger imaging studies in inception cohorts followed longitudinally are needed to address this question.

\section{References}

1. van der Linden S, Valkenburg HA, Cats A. Evaluation of diagnostic criteria for ankylosing spondylitis. A proposal for modification of the New York criteria. Arthritis Rheum. 1984;27(4):361-8.

2. Rudwaleit M, Haibel H, Baraliakos X, Listing J, Marker-Hermann $\mathrm{E}$, Zeidler H, et al. The early disease stage in axial spondylarthritis: results from the German Spondyloarthritis Inception Cohort. Arthritis Rheum. 2009;60(3):717-27.

3. Bennett AN, McGonagle D, O'Connor P, Hensor EM, Sivera F, Coates LC, et al. Severity of baseline magnetic resonance imagingevident sacroiliitis and HLA-B27 status in early inflammatory back pain predict radiographically evident ankylosing spondylitis at eight years. Arthritis Rheum. 2008;58(11):3413-8.

4. Rudwaleit M, van der Heijde D, Landewe R, Listing J, Akkoc N, Brandt J, et al. The development of assessment of SpondyloArthritis International Society classification criteria for axial spondyloarthritis (part II): validation and final selection. Ann Rheum Dis. 2009;68(6):777-83.

5. Rudwaleit M, Jurik AG, Hermann KG, Landewe R, van der Heijde $\mathrm{D}$, Baraliakos X, et al. Defining active sacroiliitis on magnetic resonance imaging (MRI) for classification of axial spondyloarthritis: a consensual approach by the ASAS/OMERACT MRI group. Ann Rheum Dis. 2009;68(10):1520-7.

6. Aydin SZ, Maksymowych WP, Bennett AN, McGonagle D, Emery $\mathrm{P}$, Marzo-Ortega $\mathrm{H}$. Validation of the ASAS criteria and definition of a positive MRI of the sacroiliac joint in an inception cohort of axial spondyloarthritis followed up for 8 years. Ann Rheum Dis. 2011;71(1):56-60.

7. Rudwaleit M, Schwarzlose S, Hilgert ES, Listing J, Braun J, Sieper J. MRI in predicting a major clinical response to anti-tumour necrosis factor treatment in ankylosing spondylitis. Ann Rheum Dis. 2008;67(9): 1276-81.

8. Bochkova AG, Levshakova AV, Bunchuk NV, Braun J. Spinal inflammation lesions as detected by magnetic resonance imaging in patients with early ankylosing spondylitis are more often observed in posterior structures of the spine. Rheumatology (Oxford). 2010;49(4):749-55.

9. Zochling J, van der Heijde D, Dougados M, Braun J. Current evidence for the management of ankylosing spondylitis: a systematic literature review for the ASAS/EULAR management recommendations in ankylosing spondylitis. Ann Rheum Dis. 2006;65 (4):423-32.

10. Rudwaleit M, Listing J, Brandt J, Braun J, Sieper J. Prediction of a major clinical response (BASDAI 50) to tumour necrosis factor alpha blockers in ankylosing spondylitis. Ann Rheum Dis. 2004;63(6):665-70.

11. Barkham N, Keen HI, Coates LC, O'Connor P, Hensor E, Fraser $\mathrm{AD}$, et al. Clinical and imaging efficacy of infliximab in HLAB27-Positive patients with magnetic resonance imagingdetermined early sacroiliitis. Arthritis Rheum. 2009;60(4):946-54.

12. Haibel H, Rudwaleit M, Listing J, Heldmann F, Wong RL, Kupper $\mathrm{H}$, et al. Efficacy of adalimumab in the treatment of axial spondylarthritis without radiographically defined sacroiliitis: results of a twelve-week randomized, double-blind, placebo-controlled trial 
followed by an open-label extension up to week fifty-two. Arthritis Rheum. 2008;58(7):1981-91.

13. Maksymowych WP, Chiowchanwisawakit P, Clare T, Pedersen SJ, Ostergaard M, Lambert RG. Inflammatory lesions of the spine on magnetic resonance imaging predict the development of new syndesmophytes in ankylosing spondylitis: evidence of a relationship between inflammation and new bone formation. Arthritis Rheum. 2009;60(1):93-102.

14. Pedersen SJ, Chiowchanwisawakit P, Lambert RG, Ostergaard M, Maksymowych WP. Resolution of inflammation following treatment of ankylosing spondylitis is associated with new bone formation. J Rheumatol. 2011;38(7):1349-54.

15. Chiowchanwisawakit P, Lambert RG, Conner-Spady B, Maksymowych WP. Focal fat lesions at vertebral corners on magnetic resonance imaging predict the development of new syndesmophytes in ankylosing spondylitis. Arthritis Rheum. 2011;63(8):2215-25.

16. Wanders A, Heijde D, Landewe R, Behier JM, Calin A, Olivieri I, et al. Nonsteroidal antiinflammatory drugs reduce radiographic progression in patients with ankylosing spondylitis: a randomized clinical trial. Arthritis Rheum. 2005;52(6):1756-65. 